EGU2020-16283

https://doi.org/10.5194/egusphere-egu2020-16283

EGU General Assembly 2020

(c) Author(s) 2020. This work is distributed under

the Creative Commons Attribution 4.0 License.

\title{
Cooling effects on induced seismicity in supercritical geothermal systems
}

\author{
Francesco Parisio ${ }^{1}$, Victor Vilarrasa ${ }^{2,3}$, Wenqing Wang ${ }^{4}$, Olaf Kolditz ${ }^{4,5}$, and Thomas Nagel ${ }^{1}$ \\ ${ }^{1}$ Chair of Soil Mechanics and Foundation Engineering, Institute of Geotechnics, Technische Universität Bergakademie \\ Freiberg, Freiberg, Germany \\ ${ }^{2}$ Institute of Environmental Assessment and Water Research (IDAEA), Spanish National Research Council (CSIC), Barcelona, \\ Spain \\ ${ }^{3}$ Associated Unit: Hydrogeology Group UPC-CSIC, Barcelona, Spain \\ ${ }^{4}$ Department of Environmental Informatics, Helmholtz Centre for Environmental Research - UFZ, Leipzig, Germany \\ ${ }^{5}$ Applied Environmental Systems Analysis, Technische Universität Dresden, Dresden, Germany
}

Geothermal energy is a fundamental piece of the puzzle in the carbon-free energy transition necessary to mitigate adverse effects of climate change. In this context, so-called supercritical geothermal systems (where resident brine is above its critical point) can reach a power output of up to $50 \mathrm{MW}$ per-well and are becoming a reality thanks to the research effort of the international community. Supercritical systems are inherently complex because of fluid and solid rheology and because of the non-linear couplings involved between pore pressure, temperature and deformation in the porous and fractured rock. In this contribution, we have performed finite element analyses of coupled thermo-hydro-mechanical (THM) conditions in a supercritical geothermal system. The formulation employed includes a porosity-dependent permeability relationship that is derived through mass balance equations of the solid skeleton. The equations of state of water are based on IAPWS standards and span the whole range of temperature and pressure of interest in supercritical geothermal systems. We have analyzed an injectionproduction doublet scenario at $5.5 \mathrm{~km}$ depth, where the two wells are spaced $500 \mathrm{~m}$ apart and a major fault lies between them. When injecting in isothermal conditions, because of water mobility of the supercritical phase is higher than the liquid phase, minor perturbations are observed in the major fault. Seismicity increases rapidly following a pressure-diffusion response and only microseismicity is expected as the stress in the major fault shows minor changes. On the contrary, cold fluid injection generates large thermal-induced stress changes that diffuse following advective heat transport laws. Micro-seismicity is quickly triggered and, within 10 years in the current setting, the perturbation reaches the $250 \mathrm{~m}$ distant fault, where larger magnitude events are possible. Our findings bear important consequences in terms of safety of supercritical geothermal systems and proved that seismic response in amplified by re-injection-induced cooling. 